\title{
ANTIBODIES AND SPERM SURVIVAL IN THE FEMALE TRACT OF THE MOUSE AND RABBIT
}

\author{
J. COHEN AND D. J. WERRETT \\ Department of Zoology and Comparative Physiology, \\ University of Birmingham, Birmingham B15 2TT
}

(Received 4th Fuly 1974)

\begin{abstract}
Summary. Rabbit and mouse spermatozoa from male and female tracts have been examined for their species-antigenic surface character, and for adherent antibodies, by double immunofluorescence techniques. Mouse spermatozoa from the ductus deferens showed an area over the acrosome which was positive to anti-mouse serum that had been absorbed with some male mouse somatic tissues including blood, but those from the uterus and oviduct were not stained. Spermatozoa from the uterus were shown to have an antibody coat on the acrosome, with anti-mouse IgG, but those from the ductus deferens and oviduct did not. Rabbit spermatozoa were more variable but their activity was similar: ejaculated spermatozoa sometimes already had antibody of male origin; the majority of the spermatozoa arriving early in the uterus were coated, but in general those that attained the oviducts were not coated.

The results are interpreted as evidence for selection by the female tract of a small antigenically different population; the majority of spermatozoa are rejected and/or destroyed.
\end{abstract}

\section{INTRODUCTION}

The destruction of the vast excess of gametes which accompanies fertilization in most animals has been amply documented, especially in mammals (review by Cohen, 1971). To some extent, this excess is removed by passive drainage of immotile spermatozoa from the female tract (Mattner \& Braden, 1969) but another major factor is phagocytosis in the uterus.

Austin (1960) noted that the acrosomes of rabbit spermatozoa were coated by IgG before or during their destruction by phagocytosis. Symons (1967), who investigated this coating by an immunofluorescence technique, concluded tentatively that dead or dying rabbit spermatozoa were coated to facilitate their location and removal by phagocytes. $\mathrm{He}$ found that washed ejaculated spermatozoa, which had been killed by alcohol, were all coated, but that about $30 \%$ of the spermatozoa in the uterus and oviducts were not coated 7 to $12 \mathrm{hr}$ after coitus; about the same proportion were motile. Swanson Beck, Edwards \& Young (1962) had already raised questions relating to isoantigenicity of spermatozoa and neither they nor Bratanov (1969) or Bedford (1970) found a satisfactory explanation. 
More recently, Johnson (1973) has suggested that, because spermatozoa are antigenic, a mechanism is required to prevent the immunization which would give the risk of sterilization of the female. A low titre of circulating anti-sperm auto-antibody might have this function, like the anti-Rh antibody injected into pregnant $\mathrm{Rh}-$ women to prevent any challenge with $\mathrm{Rh}+$ antigen from the fetus.

Johnson \& Hunter (1972) have reinvestigated the rabbit using a very specific antibody technique. They produced anti-rabbit-sperm antibody and antirabbit-sperm-coating antibody in the cow. Their experiments showed that 'sperm coating antigens' were present on the acrosomes of ejaculated spermatozoa, but only a small proportion of spermatozoa recovered from the oviducts or uterus $10 \mathrm{hr}$ later showed this coating: the majority had sperm-specific antigens. Johnson \& Hunter (1972) concluded that the uterine environment removed the sperm-coating factor, which they equated with the sperm-coating antigen of Weil \& Stefanovic (1969), as part of the process of capacitation. It was also thought possible that removal of the sperm-coating antigens exposed the 'foreign acrosomal protein' to the immunological defences of the female, resulting in phagocytosis or an initial step in capacitation. Each spermatozoon was considered to change from 'dull' and coated to 'bright' and uncoated and Johnson \& Hunter speak of removal of the coat from the acrosome of a spermatozoon. The 10-hr population of spermatozoa used by these authors was only a small fraction of the initial population, however, and another explanation is possible.

We have been investigating antibody reactions in mouse and rabbit spermatozoa in relation to antibody coating and phagocytosis. Our early results (Cohen, 1974) suggested that most spermatozoa are coated with antibody and phagocytosed but that a few are antigenically different and survive. This paper reports the investigation of the process of sperm attrition, both numerically and with well-controlled immunological procedures.

\section{MATERIALS AND METHODS}

\section{Mice}

Mice of laboratory strains $\mathrm{C} 3 \mathrm{H} / \mathrm{Mg}, \mathrm{DBA} / 1$ and $\mathrm{A} 2 \mathrm{G}$ were used. They were naturally inseminated, but some females received intraperitoneal injections of 3.5 i.u. PMSG followed $36 \mathrm{hr}$ later by 3.5 i.u. HCG and were put with the male $8 \mathrm{hr}$ after administration of HCG. The mice were allowed to mate from 8 to $14 \mathrm{hr}$ after the injection. They were killed by cervical dislocation from 2 to $12 \mathrm{hr}$ after finding a vaginal plug, and the uteri and oviducts were clamped and removed promptly. Great care was taken to avoid even slight change of temperature post mortem since this caused contractions of the myometrium and, therefore, possible mixing of uterine and oviducal contents. Uteri and oviducts were flushed separately, each with about $1 \mathrm{ml}$ phosphate-buffered $0.9 \%$ saline (PBS: Oxoid Dulbecco A). Dilutions of uterine flushings were prepared for counting in PBS; $0.02 \mathrm{ml}$ drops of the diluted flushings were allowed to dry on slides, fixed with acetic alcohol, stained in Casaret's stain (1953) and permanently mounted in DPX. All spermatozoa in three such drops were usually 
counted to estimate the number in the uterus. A known number of spermatozoa was inseminated into and subsequently flushed from a uterus and was counted in this way to validate the method. Three drops $(0.02 \mathrm{ml}$ each $)$ were also prepared from the flushings of the oviducts, but with only one dilution step in PBS, and were stained and counted similarly.

\section{Rabbits}

The rabbits used were New Zealand White does and stud bucks, obtained from Hylyne Rabbits or Cheshire Rabbit Farms; chinchilla and agouti-lop stud bucks available in the department were also used. Some were naturally mated, and some were artificially inseminated and either injected with 50 to 75 i.u. HCG at the same time or mated immediately afterwards with a vasectomized buck. Since sperm numbers vary greatly within and between bucks (Morton, 1970), artificial insemination was preferred so that the numbers of spermatozoa inseminated were known. The does were killed by deep anaesthesia induced by intravenous Nembutal followed by exsanguination from the heart. The table was electrically warmed to maintain the body temperature of the rabbit in the 2 to $3 \mathrm{~min}$ after breathing ceased and before the uterus and oviducts could be clamped. If cooling occurred, the number of spermatozoa found in the oviducts was up to twenty times higher than otherwise because the uterine contents were expelled cranially by the contracting uterus. (The three high counts not included in his means by Braden (1953) may have had similar cause.)

The uteri were flushed in situ with $2 \mathrm{ml} \mathrm{PBS}$ at $37^{\circ} \mathrm{C}$ injected and recovered through a $2 \mathrm{ml}$ syringe and 21-gauge needle. The oviducts were each filled to tautness with $37^{\circ} \mathrm{C}$ TG medium 199 (Flow Labs 1043D) through the fimbria which was then clamped. After dissection of the caudal end, the fat was rolled off with a cotton bud, and all the liquid was expressed through this end. The liquid was then forced through again by a Pasteur pipette.

Insignificant numbers $(<12)$ of spermatozoa were found in subsequent washings after centrifugation. Fixed volume counts (Cohen, 1971) were performed as described for the mouse.

\section{Labelling}

Most of the immunochemicals used were obtained from Burroughs Wellcome. The rabbit anti-mouse IgG was donated by Behringwerke AG. Several other brands did not achieve either sufficient selectivity or enough fluorescence, and consistent results could not be obtained in early trials.

Specimens were examined under a Vickers M41 microscope or Zeiss Photomicroscope 1, modified to permit simultaneous red-light phase-contrast and epi-illumination with $\mathrm{HBO} 200$ blue light (BG12), with barrier filters 50 and 65 for fluorescein, and 52 alone for rhodamine-conjugated antibody. High-speed Ektachrome, uprated to 640 ASA by Tayler Colour Laboratories, Birmingham, U.K., was used for routine photography in all tests with about 2 to $4 \mathrm{~min}$ exposure.

Species precipitating antibodies, RSOl (sheep anti-rabbit) or RRO8 (rabbit anti-mouse/rat), or rabbit anti-mouse IgG, were used as the first stage of 
the double-antibody technique. Fluorescein-conjugated rabbit anti-sheep/goat $\gamma$-globulin MF11, or fluorescein- or rhodamine-conjugated sheep anti-rabbit- $\gamma$ globulin $\mathrm{MFO} 2$ or MRO2 were used to label this first stage, and $\mathrm{MRO} 2$ or MFO2 were used to label rabbit IgG on rabbit spermatozoa directly. Antibodies were usually absorbed for at least $2 \mathrm{hr}$ at room temperature with rabbit or mouse tissues other than spermatozoa, and the conjugated antibodies used for the second stage were also absorbed with excess washed spermatozoa so that they did not stain the spermatozoa non-specifically during tests. Eight to ten drops of various suspensions (e.g. spermatozoa, liver cells, serum) were placed on a very clean microscope slide grid-marked with a writing diamond, and were allowed to dry in air. After drying, it was confirmed that each drop had remained within its grid position. The slides were fixed in anhydrous acetone (over sodium sulphate) at $-20^{\circ} \mathrm{C}$. After thorough washing with PBS, the first antibody was pipetted onto the slides to cover all the drops, and the slides were then rocked gently for $30 \mathrm{~min}$ in a humid chamber at room temperature. After another thorough wash, the second antibody treatment was applied for $30 \mathrm{~min}$ and was followed by a third wash. The slides were mounted in PBS : glycerol $(1: 4, \mathrm{v} / \mathrm{v})$. At least eight similarslides, usually thirty-six, were dealt with differently but simultaneously, giving a range of blanks and controls and ensuring uniform treatment for all the drops on one slide. Each slide carried a true negative and a true positive spot which could be compared with the experimental ones, and in each test, negative controls, antigen staining and antibody staining could be compared for the same sample, and comparisons could also be made with the antigen and antibody spots on each slide. Although cumbersome, this method worked well, giving us confidence in the specificity of our staining.

\section{EXPLANATION OF PLATE 1}

Spermatozoa with antigen or antibody shown by immunofluorescence. All samples were washed at least seven times in phosphate-buffered saline before fixation, epi-illuminated with HBO200 light through a BG12 filter and viewed with phase-contrast red light. Colour transparencies were rephotographed through a green filter so that fluorescence shows as white-grey and the phase picture as grey-black. $\times 500$.

Fig. 1. Mouse spermatozoa, uncontaminated with blood, extracted from the ductus deferens and stained for mouse antigens different from those of the absorbing tissues.

FIG. 2. Mouse spermatozoa from the oviduct on the same slide as those shown in Fig. 1, stained for mouse antigens different from those of absorbing tissue.

Fig. 3. Mouse spermatozoa flushed from the uterus $2 \mathrm{hr}$ post coitum and stained for IgG.

Fig. 4. Mouse spermatozoa from the same sample as those shown in Fig. 1, incubated with mouse serum for $30 \mathrm{~min}$ and stained for IgG on the same slide as those shown in Fig. 3.

Fig. 5. Rabbit spermatozoa from an ejaculate, incubated with rabbit serum for $30 \mathrm{~min}$ and stained for IgG for comparison with those shown in Fig. 4.

Fig. 6. Rabbit spermatozoa, of a different ejaculate from those shown in Fig. 5, stained for globulin; this buck usually produced 'coated' spermatozoa.

Frg. 7. Rabbit spermatozoa flushed from the uterus $10 \mathrm{hr}$ post coitum and stained for antibody. Note the negative spermatozoa and the positive phagocytes.

Fig. 8. Rabbit spermatozoa, from a different sample from those shown in Fig. 7, flushed from the uterus $10 \mathrm{hr}$ post coitum and stained for rabbit antigens. Note the variably positive acrosomes and the positive phagocytes. 
I'. IIt:
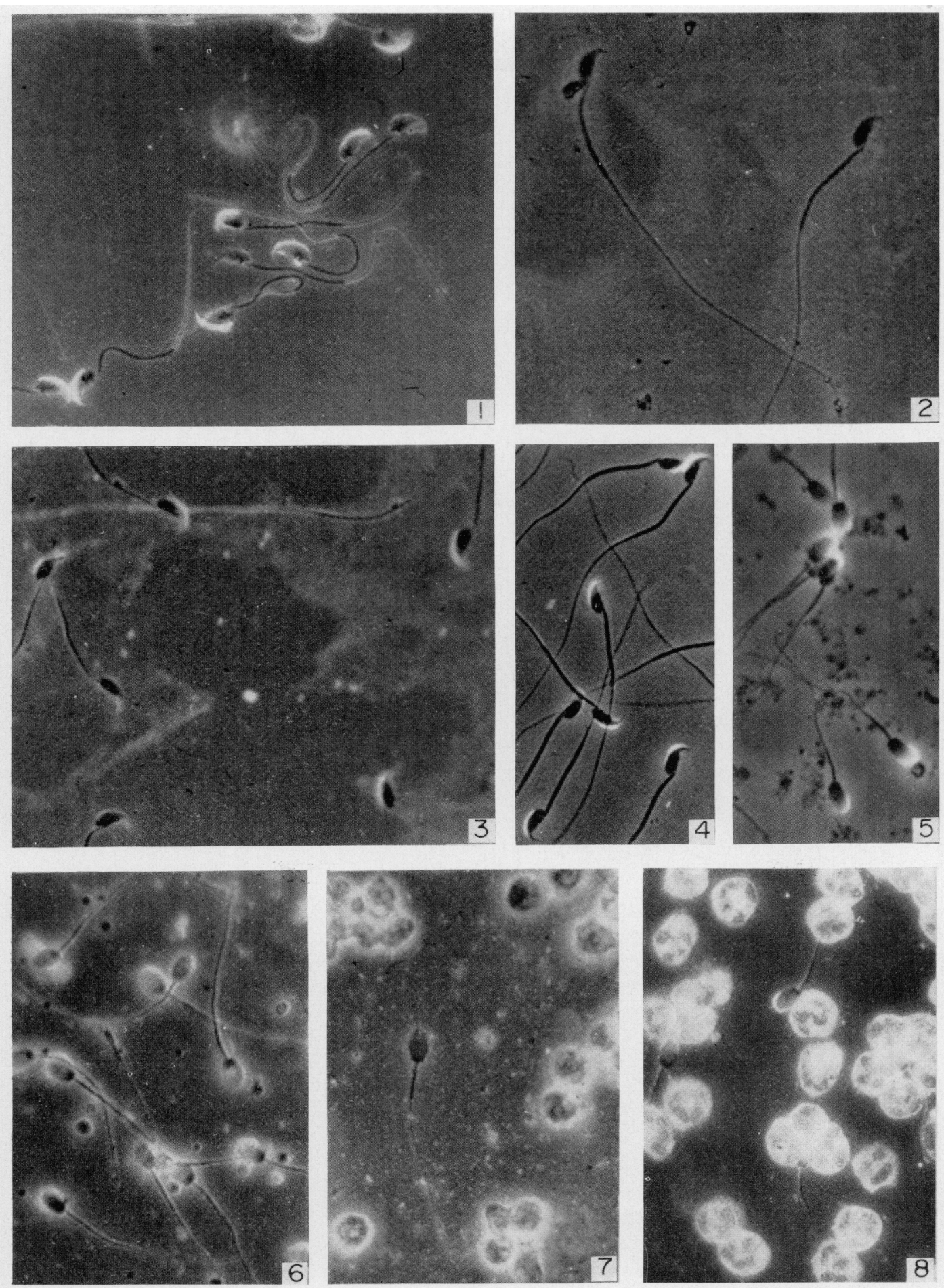

Fin 1300.80 


\section{RESULTS}

Mouse

The logarithmic mean number (expressed with the asymmetry of the variance and with the $\log$ means \pm S.E. in parentheses) of spermatozoa in the uterus $2 \mathrm{hr}$ after finding a vaginal plug was $8.04 \pm{ }_{1 \cdot 6}^{1 \cdot 3} \times 10^{6}(6.905 \pm 0.0663)$. Mean numbers in each oviduct were $4.89 \pm 2.0 \times 10^{2}$ spermatozoa $(2 \cdot 69 \pm 0 \cdot 24$.) The number of rabbit spermatozoa in the uterus about $6 \mathrm{hr}$ after insemination was $9 \cdot 8 \pm \pm_{4 \cdot 4}^{10 \cdot 2} \times$ $10^{4}(4.99 \pm 0.29)$ and the number in the oviduct was $1 \cdot 6 \pm 0.4 \times 10^{3}(3 \cdot 02 \pm 0 \cdot 22)$.

When the slides of material from the experimental mice were incubated with unabsorbed anti-mouse species antibody, followed by conjugated antibody, positive results were obtained for all smears. Liver and serum spots fluoresced brightly, while acrosomal fluorescence was the predominant feature of the spermatozoa.

When the slides of mouse material were incubated with anti-mouse species antibody which had been absorbed with male mouse somatic tissue, followed by conjugated antibody, the following results were obtained. For liver tissue, there was no specific fluorescence, only autofluorescence; all the acrosomes of spermatozoa from the ductus deferens fluoresced (Pl. 1, Fig. 1); there was no fluorescence of spermatozoa from the uterus, or from the oviducts; spermatozoa from the ductus deferens which had been washed in serum did not fluoresce; the serum spot was negative. In early tests, spermatozoa from the ductus deferens or epididymides stained patchily or not at all if there was any blood contamination. Spermatozoa from the testis were included in several early trials, and the acrosomes fluoresced brightly, frequently only as a thin 'edge', however, unless there was any contamination with blood.

When the mouse slides were incubated with anti-mouse immunoglobulin-G antibody, followed by conjugated antibody, the following results were obtained. There was no specific fluorescence, only autofluorescence of liver tissue; spermatozoa from the ductus deferens did not fluoresce; $>80 \%$ of spermatozoa from the uterus fluoresced over their acrosomes; $10 \%$ of spermatozoa from the oviducts showed fluorescence; spermatozoa from the ductus which had been washed in serum fluoresced (Pl. 1, Fig. 4). Material from the testis was very variable especially when there had been contamination with blood; mature spermatozoa were usually negative.

\section{Rabbit}

When the rabbit slides were incubated with anti-rabbit species antibody that had been absorbed with rabbit serum globulin, followed by conjugated antibody, the following results were obtained. Liver material was very fluorescent; about $50 \%$ of the washed ejaculated spermatozoa showed variably fluorescent acrosomes, the proportion depending on the buck; spermatozoa from the uterus after 4 to $6 \mathrm{hr}$ had only a few $(<10 \%)$ positive acrosomes; washed ejaculated spermatozoa that had been incubated in serum and re-washed had few positive acrosomes, but the proportions did not correlate with the proportions of spermatozoa from the uterus which had positive acrosomes on the same slide; spermatozoa from the uterus after $10 \mathrm{hr}$ were clear but variably stained (Pl. l, Fig. 8); the globulin spot was only slightly fluorescent at the edges. 
The following results were obtained when the rabbit slides were incubated with anti-rabbit-immunoglobulin which had been absorbed with washed rabbit somatic tissues, either conjugated itself or followed by conjugated antibody. The liver tissue was mostly negative; $50 \%$ of the washed ejaculated spermatozoa had positive acrosomes, but the proportions were the converse of those staining to the antigen for the anomalous bucks; $>90 \%$ of the spermatozoa from the uterus after 4 to $6 \mathrm{hr}$ had positive acrosomes, and were again associated with many phagocytes; $50 \%$ of the spermatozoa from the uterus after $10 \mathrm{hr}$ had negative acrosomes and were considerably outnumbered by phagocytes (Pl. 1, Fig. 7); 90\% of the ejaculated spermatozoa had positive acrosomes (Pl. 1, Fig. 5); $<10 \%$ of the spermatozoa from the oviducts had positive acrosomes.

\section{DISGUSSION}

Text-figure 1 shows the numbers of spermatozoa in the various immunological classes reported by Johnson \& Hunter (1972) for the rabbit, and our results for the mouse and rabbit.

The number of spermatozoa in the mouse uterus, about $8 \times 10^{6}$, is similar to that reported by Austin (1957) who found 10 to $14 \times 10^{6}$ and many more than that reported for an ejaculate by Snyder (1966). We may suppose, therefore, that there has been little reduction in numbers by $2 \mathrm{hr}$ post coitum and that these spermatozoa are representative of those in the ejaculate.

Virtually all these mouse spermatozoa, however, show IgG over the acrosomes. This could have come from seminal plasma or from uterine fluid. Spermatozoa from the ductus deferens do not show any IgG, but their acrosomes, like those of mature spermatozoa from the testis, 'stain' heavily with anti-species antibody, a property which has been lost by almost all the spermatozoa in the uterus. It is presumed that the IgG coating prevents visualization of the species antigenic sites over the acrosome. This is confirmed by the lack of staining of spermatozoa from the ductus deferens after they have been washed in serum, or contaminated with blood; an IgG coating was demonstrated on the sperm heads of such spermatozoa.

Spermatozoa from the oviducts, on the other hand, do not show IgG on their acrosomes. Whether the coating has been lost or was never present cannot be ascertained from simple observation. The latter seems more likely since immunological reactions at a membrane surface are not typically reversible in physiological conditions. For example, our routine washings in PBS do not remove antibody from the heads of uterine spermatozoa, and six washings in PBS do not remove the IgG from serum-incubated spermatozoa from the ductus deferens.

Oviducal spermatozoa did not show mouse antigen. Our tests, however, could only show sperm antigens which differed from those in the tissues used to absorb the sera. It is possible that these spermatozoa from the oviducts differ from those of the ductus, which did show sperm antigens by our methods, in having either a coat which is not antigenic or a spectrum of antigens like mouse tissues. The rest of the spermatozoa did not stain, even those from the ductus 

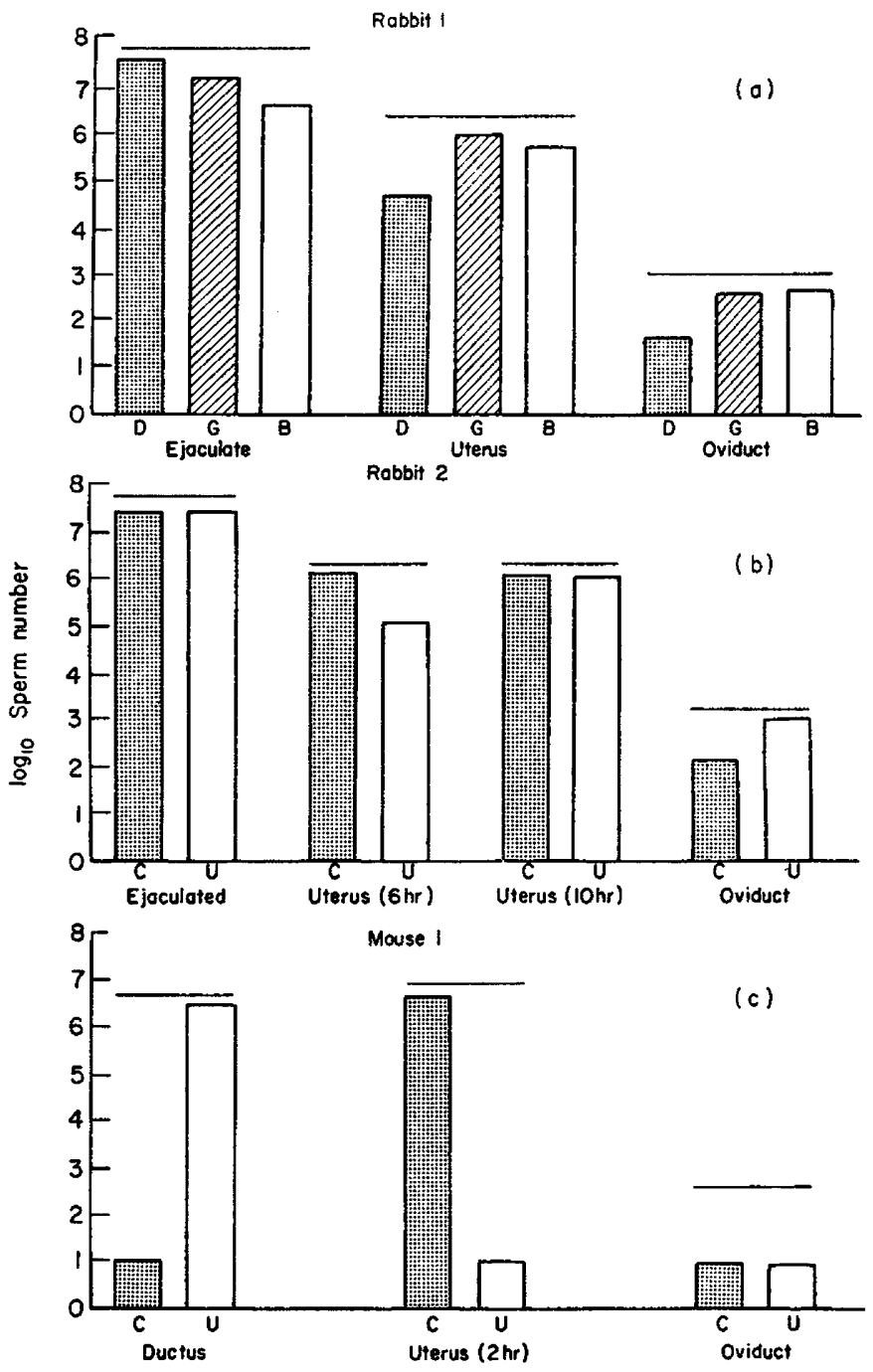

Sperm source

TEXT-FIG. 1. Antigenic properties of sperm populations in the rabbit and the mouse. Sperm numbers are given as $\log _{10}$ and the total number in each population is shown by a horizontal bar. (a) Rabbit 1, showing the results from Johnson \& Hunter (1972). Ejaculated spermatozoa and spermatozoa after $10 \mathrm{hr}$ in the uterus or $10 \mathrm{hr}$ in the oviducts were assigned to three populations: $D=$ 'dull' (coated); $G=$ 'granular' and $\mathbf{B}=$ 'bright' (uncoated). The proportion of bright spermatozoa increases, although the actual number in each population declines. Sperm numbers are from Braden (1953). (b) Rabbit 2, showing the results of the present study. One column (C) shows the number of spermatozoa coated with homologous globulin, and the other (U) shows the number of 'uncoated' spermatozoa. All ejaculated spermatozoa could be coated with serum globulin (see text). The uterine population at $6 \mathrm{hr}$ was being augmented from cervical and vaginal populations and those spermatozoa in the uterus at $10 \mathrm{hr}$ have come from a much larger temporary uterine population, thus explaining the similar size of the two populations. (c) Mouse 1 showing the results of the present study; $\mathrm{C}=$ coated spermatozoa, $\mathrm{U}=$ uncoated spermatozoa. Spermatozoa from the ductus deferens show only mouse surface antigens, although nearly all can be coated with serum globulin (see text). In the uterus, nearly all the spermatozoa were coated, but none in the oviduct was. The spermatozoa in the oviduct did not stain for mouse antigen or globulin by our methods. 
and we conclude that it too only possesses antigens which are common to our absorbing mouse tissues. It may be that the diploid nucleus-prescribed proteins are common to the sperm surface and to our absorbing tissues; the mice were from a very homozygous inbred strain and most of them were probably very similar. Haploid nucleus-prescribed protein would be expected to be similar unless such small post-meiotic synthesis as there is reflects genetic errors during spermatogenesis (Cohen, 1974).

The spermatozoa from the rabbit uterus were only a small proportion of the ejaculate, 1 to $2 \times 10^{6}$ of 6 to $20 \times 10^{7}$. They were usually outnumbered at 4 to $6 \mathrm{hr}$ post coitum by leucocytes, most of which were seen to have sperm débris inside (Bedford, 1970). The heads of these spermatozoa showed the presence of IgG but a few showed antigen staining. Unfortunately, there seemed to be no correlation between these proportions for any one sperm sample. Many ejaculated spermatozoa already had IgG; after washing in female serum, almost all had IgG and were presumably coated by uterine fluid, which contain IgG (Edwards, 1971). Since the spermatozoa in the uterus are only $1 \%$ of the ejaculate, those which have not been coated in the uterus, usually $<10 \%$, would have been about a tenth of $1 \%$ of the ejaculated spermatozoa. This minority population, which might never have been coated with IgG, would probably be undetectable in the ejaculate by our methods; we would score all spermatozoa as positive. In the oviduct of the mouse and the rabbit, at least $90 \%$ of the few spermatozoa present do not show IgG on their acrosomes. This argues that they may be a special population, selected by the tract, from the minority population in the ejaculate.

Other explanations for our results have been suggested. They include possibilities of removal of coats from the ductus spermatozoa of the mouse or ejaculated spermatozoa of the rabbit to reveal new antigens; or the removal of the plasmalemma and outer acrosome membrane to reveal the antigenicity of the acrosomal contents or inner acrosomal membrane. The similar reactivities of mature testicular spermatozoa and those from the ductus deferens suggest that there is no important coating on the latter. Transmission electron microscopy of the various sperm fractions of the mouse, together with labelling of mouse antigens and antibodies with cytochrome-c conjugates, has shown that the plasma membrane and outer acrosomal membrane are intact on most of the oviducal spermatozoa, although evidence of antibody attack is seen on many early spermatozoa in the uterus (D. J. Werrett, unpublished results).

These explanations can be applied to the results of other workers who have found dramatic changes in sperm proportions. When the smallest proportion in the initial population exceeds the whole final population in number, then transformation of individuals is not the only possibility (see Text-fig. 1).

Johnson \& Hunter (1972) found that ejaculated spermatozoa (probably about $6 \times 10^{6}$ ) were $68 \%$ dull (coated), $27 \%$ granular and $6 \%$ bright (showing rabbit antigen); i.e. 3 to $4 \times 10^{6}$ spermatozoa were 'bright' when inseminated. After $10 \mathrm{hr}$, uterine spermatozoa were $2.3 \%$ dull, $61 \%$ granular, and $36 \%$ bright. The total number in the uterus was not given, but our figures suggest that there were at most $2 \times 10^{6}$ spermatozoa; i.e. there were now only about $7 \times 10^{5}$ bright spermatozoa, $13 \times 10^{6}$ granular, and a few, $5 \times 10^{4}$, dull. Thus the 
actual numbers of 'bright' spermatozoa have declined about eightfold, although their proportion is much higher. These figures do not distinguish between transformation, the hypothesis of Johnson \& Hunter (1972) and the selective removal of coated spermatozoa which we suggest. This is also true of our figures. How, then, can we distinguish between transforming and selecting factors during capacitation? The observations of Symons (1967) favour interpretation towards the selection theory, because he found that IgG-coated spermatozoa were primarily phagocytosed; our observations support this, and we believe that the prime function of IgG attachment is the labelling of most of the sperm population for its destruction. Johnson (1973) suggested that anti-sperm autoantibodies serve to 'mop up' adventitious sperm antigen, but this explanation would not apply to the selection theory because we must presume that haploidprescribed antigen is involved in this discrimination (Cohen, 1971, 1974).

Comparison of proportions of antigenically different spermatozoa in mammalian genital tracts cannot be of use without absolute numbers, and even then several interpretations are possible. Parallel experiments, such as those of Cohen \& McNaughton (1974), along other lines are required if hypotheses are to be critically tested. Theoretical considerations suggest that, whatever occurs during spermatogenesis to make most spermatozoa vulnerable, this cannot require induced antibody production. Even in virgin and homozygous mice, most spermatozoa are coated, but a few always survive uncoated to fertilize. This highly specific discrimination between two sperm populations, whatever its detailed immunological basis, must be included in any consideration of mammalian reproductive strategy.

\section{REFERENCES}

Austrin, C. R. (1957) Fate of spermatozoa in the uterus of the mouse and rat. F. Endocr. 14, 335-343. Austis, C. R. (1960) Fate of spermatozoa in the female genital tract. F. Reprod. Fert. 1, 151-156.

BeDpord, J. M. (1970) Sperm capacitation and fertilization in mammals. Biol. Reprod. Suppl. 2, 128158.

BRADEn, A. W. H. (1953) Distribution of sperms in the female tract of the rabbit after coitus. Aust. $\mathcal{F}$. biol. Sci. 6, 693-705.

Bratanov, K. (1969) Antibodies in the reproductive process in the female. Translation from the Russian. In Immunology and Reproduction, pp. 175-189. Ed. R. G. Edwards. I.P.P.F., London.

CAsaret, G. W. (1953) A one-solution stain for spermatozoa. Stain Technol. 28, 125-127.

CoHen, J. (1971) The comparative physiology of gamete populations. Adv. comp. Physiol. Biochem. 5, 267380.

Conen, J. (1974) Gametic diversity within an ejaculate. In Functional Morphology of the Spermatozoon, Ed. B. Afzelius. Wenner-Gren, Stockholm 1974. (In press).

Cohen, J. \& MaNaughton, D. C. (1974) Spermatozoa: the probable selection of a small population by the genital tract of the female rabbit. F. Reprod. Fert. 39, 297-310.

EDWARDs, R. G. (1971) Transmission of antibodies across membranes of the reproductive tract. In Immunology and Reproduction, pp. 28-48. Ed. R. G. Edwards. I.P.P.F., London.

Johnson, M. H. (1973) Physiological mechanisms for the immunological isolation of spermatozoa. Adv. Reprod. Physiol. 6, 279-323.

Johnson, W. L. \& Hunter, A. G. (1972) Seminal antigens: their alteration in the genital tract of female rabbits and during partial in vitro capacitation with beta amylase and beta glucuronidase. Biol. Reprod. 7, 332-340.

Mattner, P. E. \& Braden, A. W. H. (1969) Comparison of the distribution of immotile sperm in the ovine cervix. Aust. F. biol. Sci. 22, 1069-1070.

Morton, D. B. (1970) On the transport of spermatozoa in the female rabbit. Ph.D. thesis, University of Liverpool.

SNYDER, R. L. (1966) Collection of mouse semen by electro-ejaculation. Anat. Rec. 155, 11-14. 
Swanson Bzck, J., EDwards, R. G. \& Young, M. R. (1962) Immune fluorescence technique and the isoantigenicity of mammalian spermatozoa. F. Reprod. Fert. 4, 103-110.

Symons, D. B. A. (1967) Reaction of spermatozoa with uterine and serum globulin determined by immunofluorescence. 7. Reprod. Fert. 14, 163-165.

WEIL, A. J. \& STEFAnovic, D. (1969) Spermatozoa-coating antigen (SCA): its persistence in the genital tract of fertile rabbits. Fert. Steril. 20, 689-692. 